\title{
Examining the Macrosystem Level of Influence on Community Health Worker Effectiveness in the State of Nebraska: A Qualitative Approach
}

\author{
Ashley Mulcahy Toney ${ }^{1,2} \cdot$ Tyler Martin $^{1} \cdot$ Sophi Sanchez ${ }^{3} \cdot$ Megan S. Kelley $^{1} \cdot$ Angela L. Palmer-Wackerly $^{3}$. \\ Virginia Chaidez ${ }^{1}$ (1)
}

Accepted: 28 January 2022 / Published online: 4 March 2022

(c) The Author(s), under exclusive licence to Springer Science+Business Media, LLC, part of Springer Nature 2022

\begin{abstract}
Community health workers (CHWs) serve as the linkage between community and providers and are stakeholders for bridging services to the public. However, integration of CHWs into health care organizations is often lacking. This study explored macrosystem level barriers faced by CHWs and their ability to do their jobs effectively. Using qualitative interviews from CHWs $(n=28)$ in Nebraska, we used an abductive approach to derive the following themes: (1) CHWs and client macrosystem barriers, (2) CHW workforce supports, and (3) macrosystem solutions for CHW workforce sustainability. Study results also found various macrosystem barriers affecting CHW workforces including immigration policies, insurance policies, funding sources, supervisor support, and obstacles for health seeking of clients. Moreover, through the lens of CHWs, results revealed the need to provide and advocate for solutions that prioritize the needs of CHWs as they continue to fill a crucial gap in community healthcare systems.
\end{abstract}

Keywords Community health worker $(\mathrm{CHW}) \cdot$ Social ecological model $\cdot$ Workforce $\cdot$ Community and public health . Health disparities

\section{Introduction}

Community health workers (CHWs) are trusted individuals within their communities who serve as linkages between the community and health resources (i.e. health care providers, insurance networks, researchers) to improve access and delivery of services to underserved communities $[1,2]$. Despite having $\mathrm{CHW}$ as an umbrella term, CHWs can have a variety of titles including promotora, interpreter, lay health advisor, health coordinator, and community health navigator. The services they provide can include interpretation, case management, health screenings, referrals, and insurance

Virginia Chaidez

vchaidez2@unl.edu

$1 \quad$ Nutrition and Health Sciences, University of NebraskaLincoln, 104A Leverton Hall, Lincoln, NE 68583-0806, USA

2 School of Public Health, UTHealth Center for Community Health Impact, The University of Texas Health Science Center at Houston, El Paso, TX, USA

3 Communication Studies, University of Nebraska-Lincoln, Lincoln, NE, USA applications. Though these roles and titles vary, this variety and their inherent connection to the community are the very elements that make CHWs vital partners in health and healthcare teams.

Chaidez and Palmer-Wackerly proposed studying CHWs in the context of the social ecological model [2]. Bronfenbrenner's Ecological Model for Human Development argues there are five interconnected layers that influence one another in the context of health behavior [3]. Within this model, the five layers consist of (a) the individual level (i.e. an individual's attitudes and beliefs), (b) the microsystem level (i.e. an individual's immediate environment like family and schools), (c) the exosystem level (i.e. an individual's larger environment like neighborhoods and organizations), (d) the macrosystem level (i.e. an individual's shared societal and cultural ideologies and values), and (e) the mesosystem which is the level that connects each level (Fig. 1). An additional and final level proposed by Bronfenbrenner is the chronosystem (i.e. the sociohistorical events and changes during an individual's lifetime) [2]. Of these, researchers provided evidence that the CHWs are the mesosystem linkages since they connect resources to individuals, work with 
Fig. 1 Social Ecological Model with CHW as Mesosystem. Adapted from Bronfenbrenner's Ecological Model for Human Development (1997). CHWs as the mesosystem link all layers of the social ecological model (i.e. individual, microsystem, exosystem and macrosystem). The individual level consists of an individual's health, selfefficacy and communication. The microsystem is the individual's immediate environment including their support groups, health fairs, and home visits with CHWs. The exosystem is where individuals receive care, interact with employers, and go to for services. The macrosystem is the individual's cultural and societal ideologies that influence the acceptance and integration of CHWs within the health care organization or values that create barriers for CHWs and clients

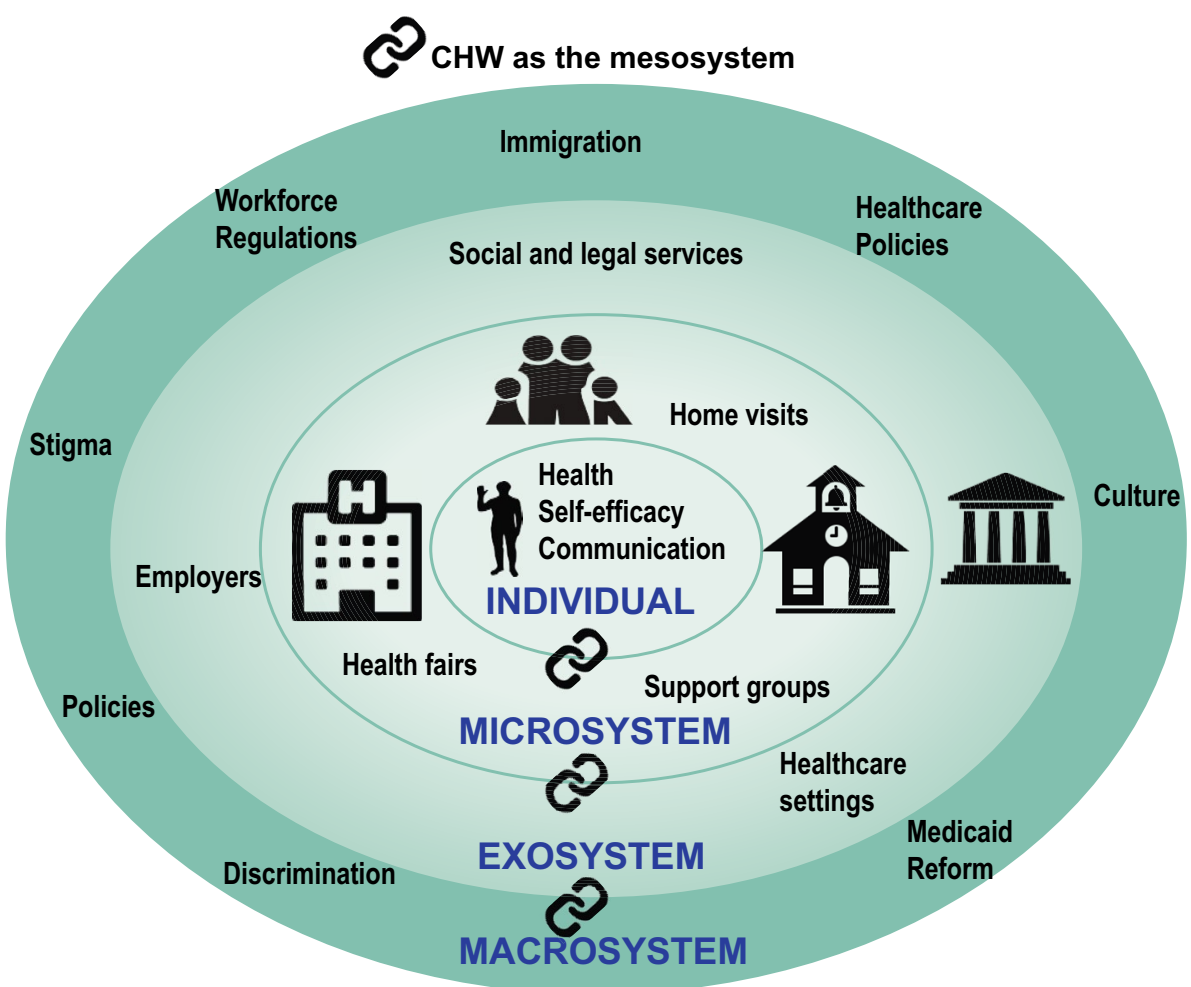

families, and serve as the liaisons between organizations or providers for the community $[2,4,5]$.

Palmer-Wackerly et al. (2020) have found CHWs have clear influence within the first three interconnected levels of health behavior. What is less clear is CHW influence at the macrosystem level and integration within healthcare organizations. This study employs an ecological approach to investigate macrosystem-level barriers to CHW effectiveness and efficacy. We propose solutions for CHW workforce sustainability and policy development surrounding integration of CHWs into healthcare infrastructure.

\section{Methods}

All procedures and interviews were approved by the institutional review board at University of Nebraska-Lincoln.

\section{Participants}

Community health workers $(\mathrm{n}=28)$ involved in communitybased and clinical settings were recruited from both rural and urban communities throughout Nebraska as described previously [5]. Recruitment was conducted through purposive sampling, "word of mouth", in-person invitations at CHW trainings, email invitations through the Nebraska Community Health Worker Association listserv, and collaboration with Nebraska's Department of Health and Human
Services. Inclusion criteria included (a) English or Spanish speaking and (b) work or volunteer experience as CHWs in Nebraska.

\section{Semi-Structured Interviews}

A semi-structured interview guide [6] was created to gain insight into CHW experiences including (a) their area of expertise and clientele, (b) their needs (for support, training, and language), (c) experience with clients' needs, stress levels, and overall health (including how client needs affect CHW mental health), and (d) thoughts on current job functions and future directions for CHWs. Researchers ensured the questions and translation of the interview guide were relevant and well received. Methods are described in detail elsewhere; briefly, Palmer-Wackerly et. al. (2020) conducted semi-structured, in-depth individual interviews with 28 CHWs in Nebraska in either English $(\mathrm{n}=12)$ or Spanish $(n=12)$ or a mix of both languages $(n=4)$ and informed consents were received [5]. Interviews were audio-recorded and transcribed verbatim, and CHWs were given pseudonyms for anonymity. Participants received a $\$ 25$ gift card for their participation.

\section{Data Analysis}

Authors used an abductive approach. This approach is a mix of deductive and inductive approaches and allowed themes 
to emerge from the interviews [7]. Eclectic coding methodology was used and is defined by Saldaña as using various coding methods (e.g., descriptive and in vivo) to categorize codes [8]. In sum, authors used five cycles of coding across approximately 2 years.

In the initial round of coding, authors (A.M.T. and T.M.) first deductively coded for all ideas that related to the different levels of the social ecological model (SEM) using numerical codes (Individual $=1$, Microsystem $=2$, Exosystem $=3$, Macrosystem $=4$, and Chronosystem $=5$ ). Mesosystem was left uncoded as we considered CHWs the "Mesosystem" in that they connect all levels together [2, 5]. For the first round of coding, A.M.T. and T.M. separately coded all interviews. V.C., A.M.T. and T.M. met regularly to compare codes. A.M.T. and T.M. initially met $85 \%$ reliability for agreement of codes. V.C. provided input to achieve $100 \%$ reliability. After providing a "preliminary code" [8], authors switched from coding for SEM levels to understanding emerging themes. Authors coded the interviews manually and created a table to include the quote, authors' interpretation of the meaning of the quote, and the designation of the social ecological level. During this cycle of coding, it became clear to coders that "CHW Barriers to Job Effectiveness" was a prominent idea found in the data. Thus, in the second round of coding, A.M.T. and T.M. inductively coded, using reflexive thematic analysis [9] to organize and identify CHW barriers and emerging solutions present within each level. Lastly, A.M.T., A.P-W. and S.S. employed a third round of coding to identify those specific barriers and solutions for macrosystem level only.

A.M.T. conducted a fourth round of coding to identify the macrosystem themes described below. The fifth and final round of coding involved categorizing subthemes within the three main parent themes discussed in the results. A.M.T. met with V.C. and A.P-W. to discuss the final coding table to reach consensus and agreement on the final codes for this manuscript to ensure reliability.

\section{Results}

Results from the interviews highlighted 3 key themes: (1) macrosystem barriers experienced by CHWs and clients, (2) macrosystem barriers affecting the CHW workforce, and (3) macrosystem solutions voiced by CHWs throughout their interviews.

\section{CHWs and Client Macrosystem Barriers}

This section specifically discusses macrosystem barriers faced by clients, which then affected CHW ability to provide effective services for them. We define macrosystem barriers as those which arise based on an "individual's shared societal and cultural beliefs, values, and ideologies" [2].

\section{Theme 1: Financial Hardships}

Insurance As part of economic and medical reform within the macrosystem sphere, insurance policies and laws affect medical coverage for those residing in the United States. Although the Affordable Care Act was enacted to increase Medicaid coverage, eligibility, and access to health care to address health disparities [10], many insurance policies still result in financial hardships and can lead participants to make difficult decisions regarding their health care. CHWs described situations in which clients forgo visiting a clinician based on cost of visit, lack of insurance, or resulting copays they may incur after the visit. CHWs often described the economic hardships faced by clients when they exceed income eligibility for Medicaid coverage. Lina, 63, works directly with parents and toddlers at a federally funded Head Start Center. She discussed how patients are unaware of payment methods available for health services once they no longer qualify for Medicaid.

... and sometimes they're taken off of is it Medicaid or Medicare? The children's program, the health insurance sometimes they are taken off for certain reasons, sometimes because of their pay, they exceed a certain income that they don't qualify anymore, so I encourage them to go ahead and go to the doctor if they really need to or their child really needs to and then ask the doctor if you can pay in payments. They should never avoid going to the doctor if it is needed.

She highlights financial hardships and lack of awareness regarding payment options may serve as a barrier for clients when seeking out health care. Other CHWs describe how clients may be hesitant to go to the doctor because of "cost of medications", being unable to afford sliding scale fees from federally qualified health centers, and the belief that they will receive low quality care due to stigma about being uninsured. Although CHWs try to connect their clients to clinics, they often felt powerless when clinics did not accept patients without insurance despite "[the clinic] working with a lot of big population with no insurance." Collectively, CHWs observed that despite the expansion of Medicaid through the Affordable Care Act, financial hardships decreased health-seeking behaviors, which further exacerbates health disparities.

Immigration Macrosystem ideologies revolving around immigration and anti-immigration rhetoric pose unique barriers for immigrants, including stigma and health care 
ineligibility, which further financial hardship and reduce access to care. In the United States, immigrants commonly join high-risk trades that involve unskilled labor with low wages and lack benefits including sick time [11]. Layered with financial stress and deportation fears, CHWs express "some people out of fear of immigration and financial reasons, they wait until their symptoms are serious before seeking out help". Moreover, immigration status is tied to insurance coverage and $\mathrm{CHWs}$ "wish the insurance was actually affordable and it reached out to everybody", including immigrants. Esperanza, 57, works in a clinic as a CHW and interpreter in central Nebraska where she interacts primarily with the migrant Latine community (i.e. a gender-neutral term for Latino/a to include individuals who may not identify with a binary gender identity and more closely associated with the Spanish language). Identifying as an immigrant, she shared the experiences faced by the migrant community including how "people that, they're professionals, they're doctors, they're counselors, they're accountants and they're working the meat pack plant here, because they don't know any English". She delves into the financial hardships uniquely faced by immigrants and said,

...as a new migrant, even though you already have a LPR [lawful permanent residents] card, but if you have less than five years in the states, you're not entitled to receive any credits. So I was helping out a gentleman, that he has cancer, he has no insurance, he was trying to get on the market, but he has only two years to be a, you know, he cannot receive any credits at all... and that's the curious thing, he can't receive SSI because before he was, was receiving, I think he was having TPS, you know, temporary protective services. So many years he worked with his social, so he has enough orders, to draw from SSI, but not enough years as a legal permanent resident to receive benefits.

Esperanza referred to the Medicaid and Children's Health Insurance Program (CHIP) criteria where LPR and green card holders need to have at least five years of residence in the United States after receiving "qualified" immigration status before they are eligible to receive benefits or have coverage, oftentimes termed as a waiting period [12]. In Nebraska and twenty other states, women and children are eligible to receive CHIP and Medicaid coverage; however, roughly $36 \%$ of immigrants residing in the United States for more than five years, especially in non-expansion states, remain uninsured which results in higher costs to treat and additional strain on the healthcare system [13]. In all, CHWs felt they are unable to do their job in helping their clients as policies surrounding immigration hindered their client's access to care.

\section{Theme 2: Community Resources}

A common theme raised by CHWs is how community resources-defined as assets or resources shared within the community which can improve quality of life or existing infrastructure-are inaccessible or hidden, resulting in individuals being unable to take advantage of these resources.

Lack of Awareness Several CHWs described how both clients' lack of awareness and inadvertent health care provider actions can impede access to community resources. CHWs have noticed there is a disconnect in knowing which resources are available and who can utilize these resources because "not everyone knows about the services that are being offered around the area." For example, Luz, 40, described how one client was decreasing her necessary dose of medications to stretch the amount of medication available.

This person who needs treatment. I said to her maybe there is a type of insurance that can help you so that you would be able to take this medication. She was very young. I said to her ... they will cover some medicine for free and she said to me 'well, I didn't know that.' And there are a lot of people who don't know that.

Other CHWs voice the need to provide transparency around what resources are available for the uninsured or referrals for follow-up treatment. Rosa, 58, revealed that clients do not receive treatment because "there needs to be a follow-up - maybe they don't receive the right information specifically because this or that, [or] you need to get yourself checked out, or to show them the resources they have... so there's lack of information". CHWs have noted in addition to clients and health care providers, CHWs themselves may be unaware of the existing infrastructure in their communities, thereby limiting their ability to do their jobs. Issa, 40, urged employers and state departments to "make sure they [CHWs] understand the, the health care of the city or state we are living in. How to utilize it cause we have families or things like ear infection and even if they have like a headache or something they just go straight to the emergency room, which is a lot, costs a lot for the state." Clients look to CHWs to navigate these resources, so lack of systems in place to help CHWs track and access resources limits their ability to help their clients.

Transportation Transportation is typically required for clients to seek help and treatments. However, transportation can become a barrier for clients when there are not systems in place for reaching specialty clinics or resources, such as clinics in other counties that may offer specialized translation services or treatments. Some CHWs indicated transpor- 
tation options were inaccessible with rules that limit their use for those who need it most. Issa said:

I mean we have this city bus but it's not working after 6 so for example if they go to a doctor appointment and they weren't seen until say 5 and then that's the last time the bus will stop so they just do not have any way to go to the doctor appointment.

Despite the availability of public transportation, its operating hours do not necessarily align with appointment times offered to clients. Another issue is policies set for transportation through insurance companies specify coverage solely for the individual going to an appointment. The bus or taxi will "only take the patient who is mom so she has to find someone to take care of the child... another example is that when one of the kids has a doctor's appointment mom can go with the child, but the other kids cannot go so she ends up staying and missing the well child appointment." Policies governing transportation, such as Medicaid coverage, inadvertently create other barriers - such as need for childcare-while trying to remedy the transportation barrier. In all, CHWs acknowledged the difficulties in accessing transportation for their clients and described their frustrations with the limits placed on transportation.

\section{CHW Workforce Supports}

In Nebraska, ongoing efforts are centered around supporting the $\mathrm{CHW}$ workforce. In this section, we discuss how direct and indirect factors related to $\mathrm{CHW}$ shortages - such as hiring limitations and workload expectations - can exacerbate CHW stress. We also explore the need for strengthening supervisor support in the $\mathrm{CHW}$ workforce to prevent burnout.

\section{Theme 1: Shortage of CHWs}

Burnout In Nebraska, CHW certification is not required; however, recent efforts have promoted standards for $\mathrm{CHW}$ workforce development [4, 14]. Despite advances in understanding ways to support the Nebraska CHW workforce-including perceived need for trainings, professional development, and gained interest in developing $\mathrm{CHW}$ competencies-CHWs described feeling overwhelmed while juggling a large caseload and multiple roles. Sebastian, 34, works in a Hispanic-serving community center that specializes in connecting newly arrived Latine individuals and their families to a variety of resources within the community that help meet basic needs. With this community need, he feels overwhelmed and requests for "having more people help you out... so that there is more people to divide up the work". CHWs discuss their frustrations with current workload expectations where they overextend themselves to help clients, noting it "takes away from our teamwork". Beth, 55, serves as a parenting coach at her local health department and most of her work consists of conducting home visits for her clients. She described the tedious process for one home visit as:

It takes like anywhere from 30 to 60 minutes to prepare for your home visit you know. And sometimes longer if you have an activity. You've got to get all the stuff for the activity and you know you've got to get everything together first. Then you go do your home visit which is usually 25 minutes to an hour. Then you come back and then you've got to document and that can take another hour. So totally, you're looking at 3 to 3.5 hours for one person just to do a home visit.

Beth provided a glimpse into the daily workload for a CHW conducting home visits, which is very demanding. Additionally, another CHW suggested "if we were fully staffed, I think we would be able to provide a better quality care to [clients]." CHWs collectively voice that the shortage of staff, increased workload and caseloads, and overtime makes it difficult to provide quality care to their clients.

\section{Theme 2: Supervisor Support}

The majority of CHWs stated their supervisors were supportive of trainings and encouraged CHWs to pursue professional development. Examples include supervisors being accommodating, encouraging ongoing college classes and trainings, and meeting regularly with their $\mathrm{CHW}$ employees; however, other CHWs described their supervisors as being absent or new due to turnover within their organization, having poor communication skills, and feeling they were "not on the same team". Ideally, a supportive supervisor provides mentorship, feedback, and protection of their employees by setting boundaries and advocating for them [15]. Supervisors set the tone for the workplace and provide guidance for CHWs. After working as a CHW for fifteen years, Beth described a situation where her supervisor enforced an incentive program.

I would prefer not to have those kind of incentives that have stipulations on them. If you're going to give an incentive for someone that does well and I do think that that's good. But you know like every three months but I don't think it should have a negative upon it like you're going to be wrote up and after three or four write ups, you're fired. You have no control over if the family's going to meet with you, if the family goes out of town for vacation, when the family's sick, when the family is actively using and will dodge you, you know [laugh]--. 
Beth described a situation where the supervisor believed the incentive program would boost work success among the CHWs, but instead it inadvertently added to CHW stress.

\section{Macrosystem Solutions}

The majority of CHWs offered suggestions throughout the interviews on ways to support CHWs, including the following macrosystem changes: more resources (i.e. community resources, translations, transportation) and funding for programs, higher pay and compensation, reimbursement from insurance companies for their services, streamlined resources among organizations, fluid referral systems, networking with other CHWs, and supervisor support. This section will highlight supervisor support, CHW unity, and placing value in CHWs as partners and connections as potential solutions for macrosystem barriers affecting CHWs.

\section{Solution 1: Amplifying Supervisor Support}

Listening and Offering Advice Supervisors can provide support by listening to CHWs, assisting in problem solving, and thereby alleviating some of their stress. CHWs know that their work is difficult and emotionally ridden. As Claudia said,

... if something's stressing me I usually go to her [supervisor] and just let her know what's going on.

So, we have that um, just that good relationship and I think just talking about what's going on that usually helps me with my stress levels.

Her supervisor helped her remain grounded and resilient by providing a space to debrief and tools to cope with the challenges she faces. Though not all supervisors may have the tools to help their CHWs cope with stress, they can steer their employees towards resources such as counselors, trainings for mental health, and other wellness resources.

Support and Trust Effective supervisors have trust in their CHWs, which builds their self-efficacy. They also accommodate the needs of their employees so CHWs can meet the needs of their clients. One CHW described how her supervisor helped in problem solving to find a center for her clients, which increased client access to resources. Another CHW said her supervisor "knows what I have to do, and I know what I have to do to get that done and she doesn't say anything negative to me... because she's built that trust in me." For complex cases, supervisors could provide support by stepping in and assisting CHWs. One CHW said "receiving more support on behalf of the supervisors so if there was something that we couldn't do, and they did the follow-up and if they could help them more that would be better for the client."

\section{Solution 2: Unifying CHWs}

CHWs advocated for a unified CHW workforce to "work together in harmony" and to have "more unity between community health workers". Enhancing networks among CHWs allows for sharing of knowledge and transparency of resources available. Esperanza, 57, has a vision for CHW unification where she explained "we need to have, almost all of us to have the same knowledge. What we can do to be almost of the same level." Networking combined with professional development opportunities would enhance connections between CHWs to support each other, connect around shared competencies, and navigate resources within their communities.

\section{Solution 3: Value in Partnerships and Connections}

CHWs at the Table When discussing policies and integration of CHWs into health systems and communities, legislators and organizations should involve CHWs and provide them a seat at the table because they have a wealth of information about the realities clients face when it comes to addressing their health and health care needs. Karen, 62, is a faith based CHW located in Omaha, Nebraska and has served clients for over 24 years. In response to what she would like to see for CHWs, she said.

That we have - we can be of value. It needs to be - I truly believe in an integrated service system as you all know. And wraparound services for families. And that's - and I think the community health workers play - will play a major role... But more of the wraparound more - an integrated service model, how do we involve community health workers in - in that decision making process.

CHWs can advocate for themselves and provide suggestions towards policies that shape their workforce, their integration within service models, and their capacity to provide defined services, such as interpretation or cultural navigation, that are within their scope of practice.

Connections Several CHWs voiced the need to meet clients where they are comfortable and create a connection with clients. For example, one CHW saw the need for specialized patient care where clinic staff and clinicians can use CHWs for home visits and follow-up appointments to increase access. Delores explained,

When it's feasible for them and comfortable for them. Sometimes it's very intimidating for parents to come back here where the doctor is and nurses and they're uncomfortable. If I bring the study to where they're 
comfortable with, in a comfortable setting in the church center where all the Mexicanos meet and they know their, that place and it's a, a comfortable setting for them. It has to be a setting that they're comfortable in and they have to be around people that they're comfortable with.

Immigration policy and sentiments during this time made it difficult for migrant workers and newly arrived immigrants to seek out resources for fear of deportation. With the dehumanization of undocumented immigrants and raids occurring in places that were regarded as community resources, such as clinics, CHWs knew that providing access to care for their clients was best in places where they felt safe $[16,17]$.

\section{Discussion}

In this study, we provide detailed examples of macrosystem barriers faced by CHWs including immigration and insurance policies which may inhibit direct health seeking of clients, further limiting the effectiveness of CHW assistance. Through the lens of these CHWs, they provide data that illustrate $\mathrm{CHWs}$ become limited in their effectiveness due to current unstable workforce structures. Lastly, we also voice solutions towards increasing macrosystem-level change to help CHWs do their jobs effectively.

Recommendations are suggested for employers and stakeholders to place value on the CHW role. Rosenthal and colleagues call for amplifying $\mathrm{CHW}$ voices through a community-based participatory approach that requires CHWs present as equal partners alongside researchers to influence $\mathrm{CHW}$ practices and future policies [18]. By taking this approach, CHWs are working towards informing competencies and skills which have been difficult to define since CHWs go by different names, reside in different organizations, come from different backgrounds, and may be volunteers or paid. Additionally, integration of CHWs at the table with top-level management lets CHWs advocate for their needs [18]. Several frameworks promote $\mathrm{CHW}$ roles and skills including advocacy, specializations of roles and skills, and details surrounding CHW trainings [18-22]. For example, one workforce framework delineates 3 categories of CHWs based on training, workplace setting, and scope of practice while linking these categories to measurable competencies [19]. This can aid in establishing clear workforce metrics including job descriptions, target tasks, evaluating performance, setting boundaries for CHWs and their roles to protect their time, and identifying gaps in training [19]. As the field advances, it is clear there is not a "one size fits all" and rules may be unique depending on the organization, community needs, or state needs.
Supervisors can advocate for several macrosystem supports including prioritizing CHW mental health and addressing the stigma around requesting help, providing a safe space for CHWs to discuss their needs and stress, reinforcing workload boundaries for $\mathrm{CHWs}$, and regularly meeting with their CHWs to continually strengthen their organization. Oftentimes, individuals are placed into supervisor positions without formal leadership training or the tools needed to successfully supervise a team of CHWs [21]. Development of supportive CHW supervisors will require the integration of specialized training on how to best support and meet the needs of the CHW workforce. The nature of CHW work can be emotionally draining and each encounter can require different skills; trained supervisors can more effectively coach their $\mathrm{CHWs}$ about these encounters and be a source of support for them [15]. Furthermore, proper evaluation metrics for supervisors are needed. Agarwal et al. recommend CHW supervisors use the Perceived Supervision Scale to assess CHW supervision and provide feedback on the quality of supervision [23, 24]. Lastly, CHW supervisors can use their power to advocate for their CHWs and remind stakeholders of CHW roles and requirements [25].

Our results indicate $\mathrm{CHWs}$ would rather spend time enhancing knowledge of available resources than recreating existing educational materials, translating documents, or navigating other communities and networks to find resources for their clients. In Nebraska for example, the Nebraska Resource and Referral System (NRRS) and MyLink app (formerly known as MyLNK) were created to help users find information about resources such as food, legal aid, lowto-no cost medical assistance, housing, and more [26, 27]. The NRRS was created with Nebraska CHWs in mind as a means to make resources available to Nebraskan families and provide a 24-h accessibility to this resource network [27]. Additionally, the MyLink app has been translated in several locally-representative languages including Arabic, Spanish, Somali, and Vietnamese to increase access [26]. These apps serve as examples of an "integrated resource network", as suggested by our interviewed CHWs, that enable CHWs to find existing resources so they can maximize their time with clients.

In conclusion, our study highlights macrosystem level barriers that inhibit $\mathrm{CHW}$ srom doing their jobs to their full potential. These results highlight how $\mathrm{CHW}$ sersevere despite these challenges, but also bring attention to their needs, including better workforce supports, more opportunities to unify CHWs in Nebraska, and improved mechanisms for streamlining resources. CHWs are the linkage between community and providers. It is time to mobilize buy-in from a variety of stakeholders to promote this valuable workforce, and to develop sustainable funding mechanisms to support their integration into existing systems. 


\section{Limitations}

Several limitations are present in this study. First, study participation was limited to only CHWs in Nebraska; however, researchers prioritized sampling from various settings to gain an array of participant characteristics and experiences [5]. Secondly, purposive sampling was used, which may have limited the response and reach of a representative sample. Additionally, this study was cross-sectional and may not be representative of the ongoing restructuring of CHW policies on the national level or ever evolving CHW workforce supports in Nebraska.

Further research is warranted in assessing the CHW workforce, especially applications for increased CHW supervisor training, evaluation of supervisors, and support for supervisors and CHWs in their team. Moreover, new barriers may have emerged through the novel coronavirus pandemic (COVID-19) in 2020 as the pandemic further exacerbated health disparities [28, 29]. Future studies should explore new barriers CHWs may have faced during the pandemic as they served as first responders through contact tracing, advocating for their communities, and accessing resources for their clients [28]. As the nation and Nebraska recover from the pandemic, it is possible the role of CHWs has expanded. It is imperative now more than ever CHWs be able to easily access the resources and support they need and deserve.

Acknowledgements The authors thank the CHWs for participating in their study; and the Bureau of Sociological Research (BOSR) at UNL.

Funding This study was funded by the USDA NIFA Hatch funding program through the Nebraska Agricultural Experiment Station (Accession Number 1011764) and the Social and Behavioral Science Research Consortium at the University of Nebraska-Lincoln.

\section{Declarations}

Conflict of interest The authors have no conflict of interest.

Ethical Approval The Institutional Review Board (IRB) approval for this research was received.

Consent to Participate Informed consent was received from each participant surveyed in either English or Spanish.

Consent for Publication All authors provide full consent for publication.

\section{References}

1. APHA. Policy Number 20091: Support for community health workers to increase health access and to reduce health inequities. 2009. https://www.apha.org/policies-and-advocacy/publichealth-policy-statements/policy-database/2014/07/09/14/19/suppo rt-for-community-health-workers-to-increase-health-access-andto-reduce-health-inequities. Accessed June 2021.
2. Chaidez V, Palmer-Wackerly AL. Community Health Workers: One Person Can Make a Difference. In: Dombrowski K, editor. Reducing Health Disparities: Research Updates from the field: Nebraska: Syron Design; 2016.

3. Bronfenbrenner, U. (1977). Toward an experimental ecology of human development. American Psychologist., 32(7), 513.

4. Chaidez, V., Palmer-Wackerly, A. L., \& Trout, K. E. (2018). Community health worker employer survey: perspectives on CHW workforce development in the midwest. Journal of Community Health., 43(6), 1145-1154. https://doi.org/10.1007/ s10900-018-0533-x

5. Palmer-Wackerly, A. L., Chaidez, V., Wayment, C., Baker, J., Adams, A., \& Wheeler, L. A. (2020). Listening to the voices of community health workers: a multilevel, culture-centered approach to overcoming structural barriers in US Latinx communities. Qualitative Health Research., 30(3), 423-436. https:// doi.org/10.1177/1049732319855963

6. Creswell, J. W., \& Poth, C. N. (2018). Qualitative inquiry \& research design: Choosing among approaches (4th ed.). Thousand oaks, CA: Sage.

7. Deterding, N. M., \& Waters, M. C. (2021). Flexible coding of indepth interviews: A twenty-first-century approach. Sociological Methods \& Research., 50(2), 708-739.

8. Saldaña, J. M. (2015). The Coding Manual for Qualitative Researchers (3rd ed.). SAGE Publications.

9. Braun, V., \& Clarke, V. (2019). Reflecting on reflexive thematic analysis. Qualitative Research in Sport, Exercise and Health., 11(4), 589-597.

10. Mazurenko, O., Balio, C. P., Agarwal, R., Carroll, A. E., \& Menachemi, N. (2018). The effects of medicaid expansion under the ACA: A systematic review. Health Aff (Millwood)., 37(6), 944950. https://doi.org/10.1377/hlthaff.2017.1491

11. Fernández-Esquer, M. E., Agoff, M. C., \& Leal, I. M. (2017). Living sin papeles: Undocumented Latino workers negotiating life in "illegality." Hispanic Journal of Behavioral Sciences., 39(1), 3-18.

12. CMMS. Medicaid and CHIP Coverage of Lawfully Residing Children and Pregnant Women. Centers for Medicare \& Medicaid, Medicaid.gov. 2019. https://www.medicaid.gov/medicaid/enrol lment-strategies/medicaid-and-chip-coverage-lawfully-residingchildren-pregnant-women. 2021.

13. Tolbert J, Orgera K, Singer N, Damico A. Key facts about the uninsured population. Kaiser Family Foundation. 2019; 13.

14. Su D, Toure DM, Ern J, Vinton V, Ouattara B, Crum A. Strengthening the Community Health Worker Workforce to Improve Maternal and Child Health in Nebraska: A State-Wide Assessment of Needs, Barriers, and Assets: University of Nebraska Medical Center, Center for Reducing Health Disparities CoPH;2020.

15. Brown, O., Kangovi, S., Wiggins, N., \& Alvarado, C. S. (2020). Supervision strategies and community health worker effectiveness in health care settings. NAM Perspectives. https://doi.org/10. $31478 / 202003 \mathrm{c}$

16. Lopez, W. D., Kruger, D. J., Delva, J., et al. (2017). Health implications of an immigration raid: findings from a Latino community in the midwestern United States. Journal of Immigrant and Minority Health., 19(3), 702-708. https://doi.org/10.1007/ s10903-016-0390-6

17. Lopez, M. M., \& Holmes, S. M. (2020). Raids on immigrant communities during the pandemic threaten the country's public health. American Journal of Public Health., 110(7), 958-959. https://doi. org/10.2105/ajph.2020.305704

18. Rosenthal, E. L., Fox, D. J., St. John JA, et al. (2021). The community health worker core consensus (C3) project story: confirming the core roles and skills of community health workers. In J. A. St. John, S. L. Mayfield-Johnson, \& W. D. Hernández-Gordon (Eds.), Promoting the health of the community: community health 
workers describing their roles, competencies, and practice (pp. 11-35). Cham: Springer International Publishing.

19. Covert, H., Sherman, M., Miner, K., \& Lichtveld, M. (2019). Core competencies and a workforce framework for community health workers: A model for advancing the profession. American Journal of Public Health., 109(2), 320-327. https://doi.org/10.2105/ajph. 2018.304737

20. Sabo, S., Ingram, M., Reinschmidt, K. M., et al. (2013). Predictors and a framework for fostering community advocacy as a community health worker core function to eliminate health disparities. American Journal of Public Health., 103(7), e67-e73. https://doi. org/10.2105/AJPH.2012.301108

21. Allen, C. G., Brownstein, J. N., Cole, M., Hirsch, G., Williamson, S., \& Rosenthal, E. L. (2018). Building a framework for community health worker skills proficiency assessment to support ongoing professional development. The Journal of Ambulatory Care Management., 41(4), 298-307.

22. O’Brien, M. J., Squires, A. P., Bixby, R. A., \& Larson, S. C. (2009). Role development of community health workers: An examination of selection and training processes in the intervention literature. American Journal of Preventive Medicine., 37, S262-S269. https://doi.org/10.1016/j.amepre.2009.08.011

23. Agarwal, S., Sripad, P., Johnson, C., et al. (2019). A conceptual framework for measuring community health workforce performance within primary health care systems. Human Resources for Health., 17(1), 86. https://doi.org/10.1186/s12960-019-0422-0

24. Vallières, F., Hyland, P., McAuliffe, E., et al. (2018). A new tool to measure approaches to supervision from the perspective of community health workers: A prospective, longitudinal, validation study in seven countries. BMC health services research., 18(1), $1-8$.

25. Whiteman, L. N., Gibbons, M. C., Smith, W. R., \& Stewart, R. W. (2016). Top 10 things you need to know to run community health worker programs: Lessons learned in the field. Southern Medical Journal, 109(9), 579-582. https://doi.org/10.14423/SMJ.00000 00000000504

26. MyLink LLC. (2021). MyLinkWeb. Retrieved January 2021, from https://lincolnne.mylnk.app/categories?language $=$ en

27. Lewis, C. (2021). Nebraska resource and referral system. Retrieved January 2021, from https://nrrs.ne.gov/

28. Rahman, R., Ross, A., \& Pinto, R. (2021). The critical importance of community health workers as first responders to COVID-19 in USA. Health Promotion International. https://doi.org/10.1093/ heapro/daab008

29. Goldfield, N. I., Crittenden, R., Fox, D., McDonough, J., Nichols, L., \& Rosenthal, E. L. (2020). COVID-19 crisis creates opportunities for community-centered population health: community health workers: At the center. The Journal of Ambulatory Care Management., 43(3), 184-190.

Publisher's Note Springer Nature remains neutral with regard to jurisdictional claims in published maps and institutional affiliations. 